\title{
Adaptive Antenna Composed of Six Dipole Elements for Wireless LAN - Part 2 -
}

\author{
Mitsuo Taguchi ${ }^{(1)}$, Tetsuya Yamashita* ${ }^{(2)}$
}

(1) Dept. of Electrical \& Electric Eng., Nagasaki University

(2) Graduate School of Science and Technology, Nagasaki University

1-14 Bunkyo-machi, Nagasaki-shi, 852-8521, Japan

E-mail: (1)mtaguchi@nagasaki-u.ac.jp,(2)d708184j@cc.nagasaki-u.ac.jp

\section{Introduction}

The authors have analyzed the adaptive antenna composed of six printed dipole elements for $2.4 \mathrm{GHz}$ band wireless LAN [1]. Dipole elements are radially located and fed by parallel line printed on both sides of the dielectric substrate. The radiation pattern of this antenna is controlled by turning on/off switching diodes connected near feed points of six dipoles [2]. Since six dipole elements are connected in parallel, however, the input impedance of this antenna varies depending on the number of excited elements. Therefore, the actual gain deteriorates when two or more elements are excited. The authors have shown that the input impedance characteristics can be improved by loading the varactor diode to the feed point in parallel by the numerical analysis [3].

In this paper, the adaptive antenna composed of six printed dipole elements loaded with the chip capacitor at the feed point instead of the varactor diode is numerically and experimentally analyzed. The operating frequencies of Wireless LAN are from $2.4 \mathrm{GHz}$ to $2.4835 \mathrm{GHz}$ [4]. In the numerical analysis, the electromagnetic simulator "WIPL-D" based on the method of moment is used [5].

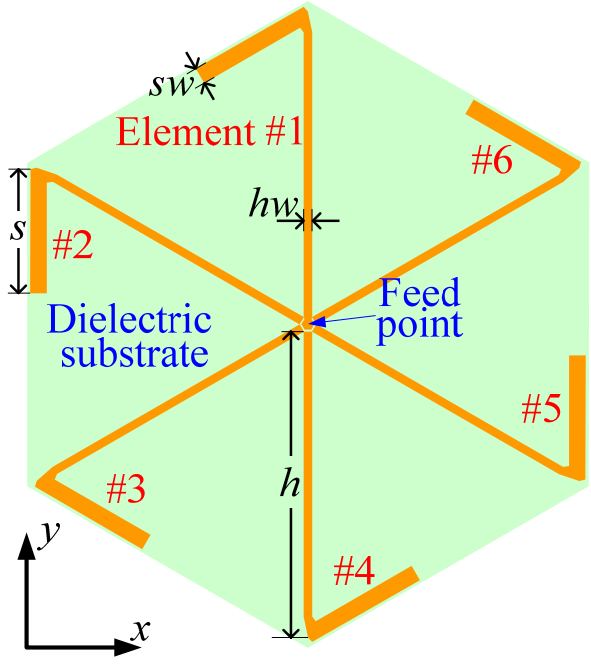

(a) Top view

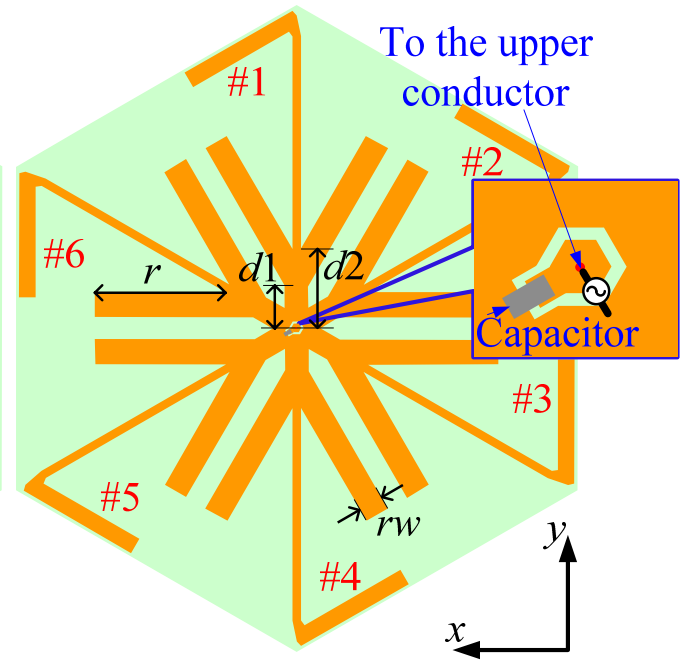

(b) Bottom view

Figure 1 Structure of antenna. $s=33.7 \mathrm{~mm}, \mathrm{sw}=7.5 \mathrm{~mm}, h=73.9 \mathrm{~mm}, h w=1.5 \mathrm{~mm}$, $r=30.5 \mathrm{~mm}, r w=7.6 \mathrm{~mm}, d 1=8 \mathrm{~mm}$ and $d 2=21 \mathrm{~mm}$. 


\section{Structure of the antenna and analytical conditions}

Figure 1 shows the structure of the antenna composed of six printed dipole elements. The dipole elements are fed by the parallel line printed on both sides of the dielectric layer with thickness of $0.8 \mathrm{~mm}$. The relative permittivity and the loss tangent of dielectric material are 2.6 and 0.0018 , respectively. At the root of parallel line on the backside of dielectric layer, the V-shaped reflector is printed. The chip capacitor is connected at feed point in parallel. The chip capacitor used is GQM series of Murata Manufacturing Co., Ltd. [6]. The tolerance of the capacitance is $\pm 0.25 \mathrm{pF}$. The total number of combination of radiating elements is 63. Figure 2 show 6 antenna patterns analyzed in this paper.
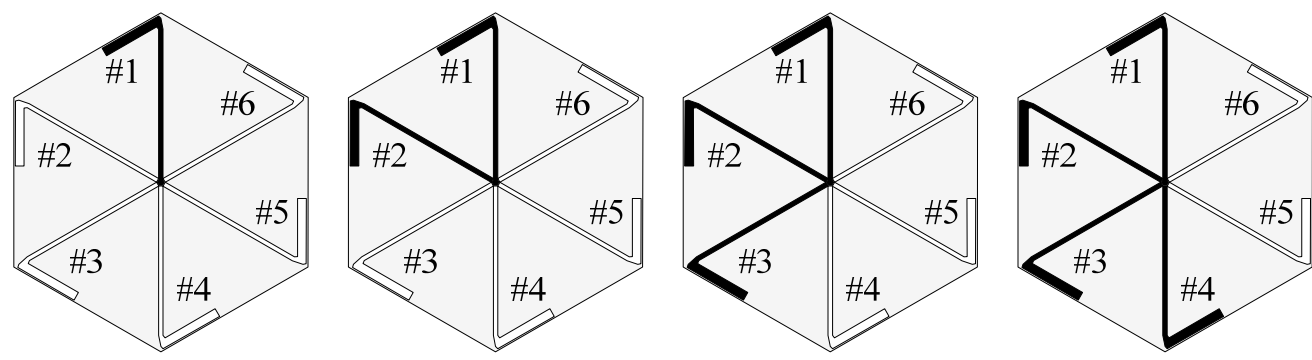

(a) Excited element \#1

(b) \#1 and 2

(c) \#1-3

(d) \#1-4

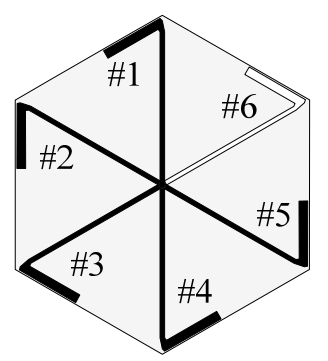

(e) \#1-5

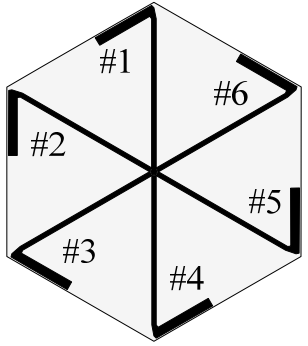

$(f) \# 1-6$
Black colored elements: ON

White colored elements: OFF

Figure 2 Analyzed antenna patterns.

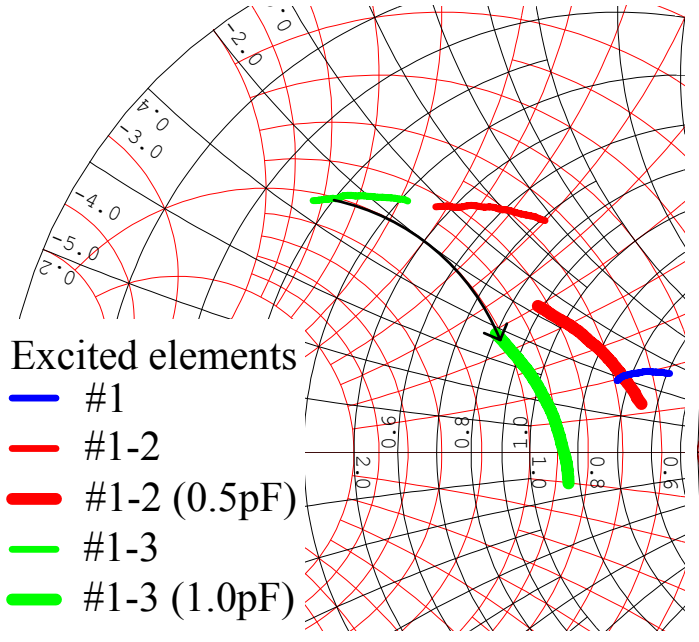

(a) Excite elements \#1, \#1 and 2, \#1-3

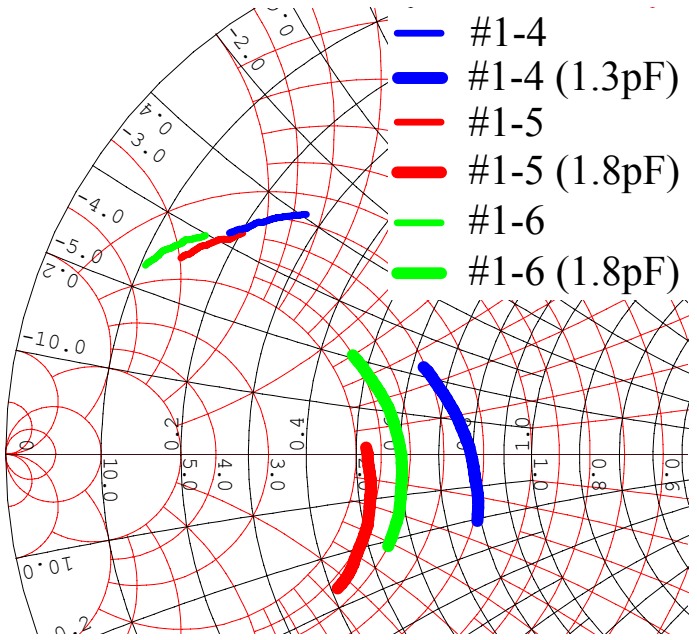

(b) \#1-4, \#1-5, \#1-6

Figure 3 Measured input impedance characteristics at $2.4 \mathrm{GHz}$ to $2.5 \mathrm{GHz}$. 


\section{Numerical and experimental results}

Figure 3 shows the calculated input impedance characteristics of the antenna with and without the capacitor. The value within parentheses of legend means the capacitance of the capacitor. The input susceptance can be changed by loading the capacitor.

Figure 4 shows the calculated and measured return loss characteristics of the antenna. In all cases, the return loss characteristics at the frequency from $2.4 \mathrm{GHz}$ to $2.4835 \mathrm{GHz}$ are improved by loading the capacitor.

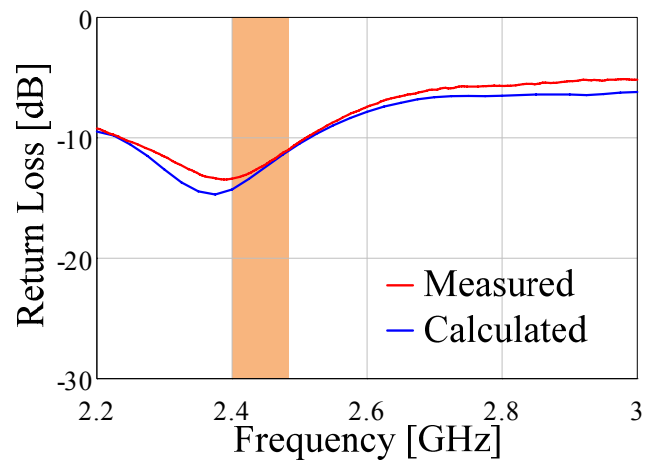

(a) Excite element \#1

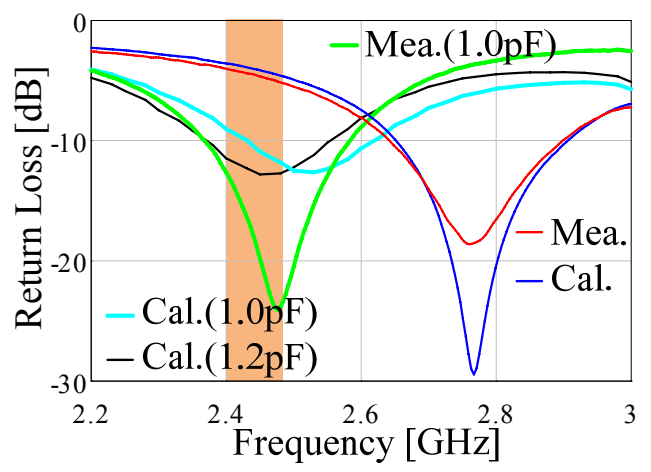

(c) $\# 1-3$

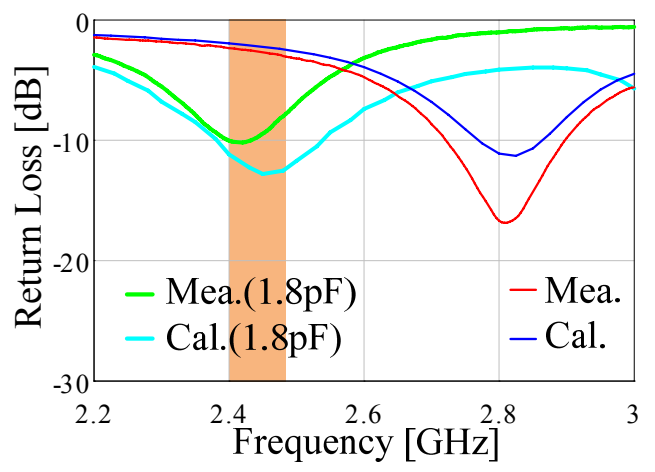

(e) \#1-5

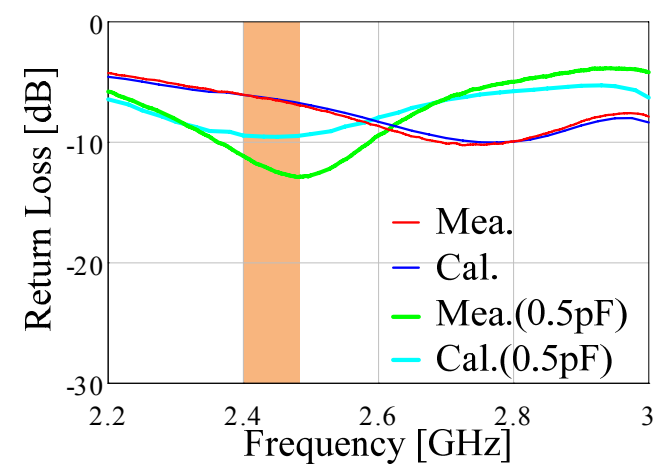

(b) \#1 and 2

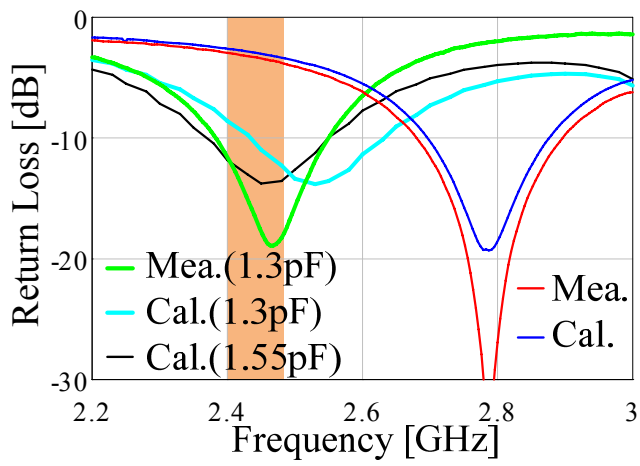

(d) \#1-4

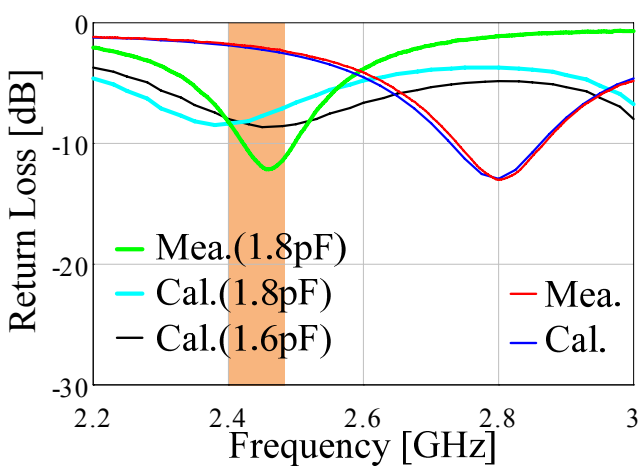

(f) \#1-6

Figure 4 Return loss characteristics. 
— Without chip capacitor - With chip capacitor
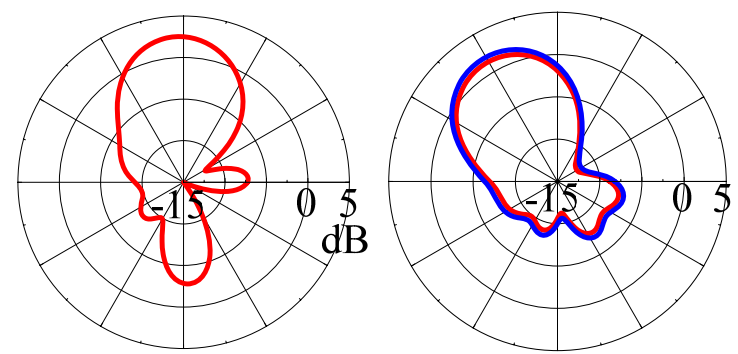

(b) \#1 and 2

$(0.5 \mathrm{pF})$

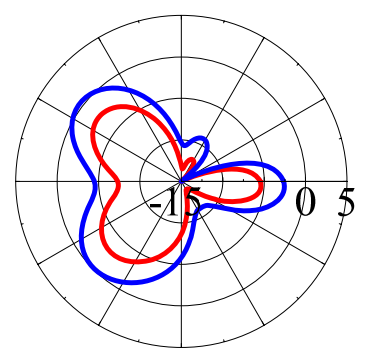

(d) \#1-4

$(1.55 \mathrm{pF})$

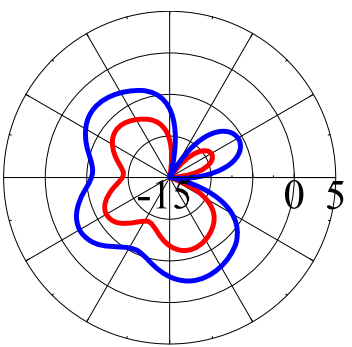

(e) \#1-5

$(1.8 \mathrm{pF})$

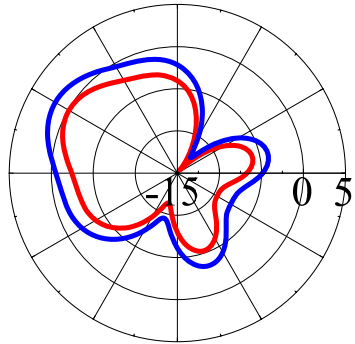

(c) \#1-3

$(1.2 \mathrm{pF})$

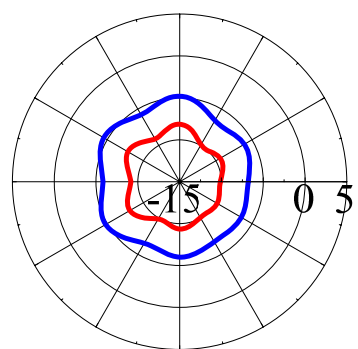

(f) \#1-6

$(1.6 \mathrm{pF})$

Figure 5 Electric field radiation pattern at $2.45 \mathrm{GHz}$

Figure 5 show the electric field radiation patterns in the xy plane. The amplitude of radiation patterns are indicated by the actual gain. The actual gain is improved by loading the capacitor.

\section{Conclusion}

In this paper, the adaptive antenna composed of six printed dipole elements with the chip capacitor has been analyzed numerically and experimentally. The input impedance characteristics are improved by the chip capacitor loaded at the feed point when the excited dipole elements are two or more.

In the next step, the antenna loaded with the varactor diode will be measured.

\section{References}

[1] M. Taguchi, T. Yamashita, D. Iwanaka, "Study on antenna of 6 dipole elements for wireless LAN" ITE Technical Report, pp. 13-16, Jan. 2008 (in Japanese).

[2] http://www.ruckuswireless.com/

[3] M. Taguchi, T. Yamashita, "Adaptive Antenna Composed of Six dipole Elements for Wireless LAN,” Proc. of ISAP, 1645414, Oct. 2008.

[4] Wi-Fi Alliance; http://www.wi-fi.org/wifi-protected-setup

[5] http://www.wipl-d.com/

[6] http://www.murata.co.jp/ 